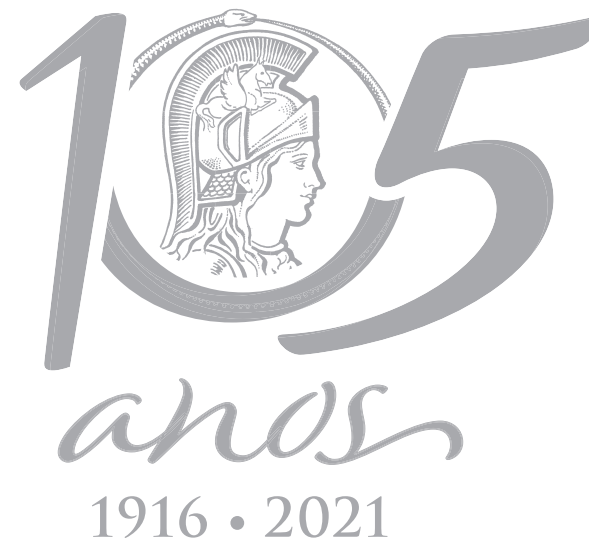

$1916 \cdot 2021$

\title{
ECOSYSTEMS
}

\section{Sea anemones (Cnidaria, Anthozoa, Actiniaria) in high sedimentation environments influenced by the Magdalena River (Colombian Caribbean)}

\author{
JEFERSON DURÁN-FUENTES, ADRIANA GRACIA \& RICARDO GONZÁLEZ-MUÑOZ
}

\begin{abstract}
Fifteen species of sea anemones (Cnidaria, Actiniaria) have been recorded so far in the Colombian Caribbean, comprising approximately $28 \%$ of the total number of known species in the Caribbean Sea. Most species recorded are associated with coral reef communities. However, in the region, no records of sea anemones are known from areas with high sedimentation conditions as is characteristic along the coastline of the Atlántico Department, in Colombia. In this area, organisms are exposed to a high degree of turbidity and sedimentation $\left(143.9 \times 10^{6} \mathrm{t}_{\text {year }}{ }^{-1}\right)$ as a result of their proximity to the Magdalena River mouth and the 26 micro-basins that flow along its coastline. Several observations and collections were made on soft bottoms, rocky, and artificial substrates in the sectors of Puerto Velero and Caño Dulce to determine the fauna that exist under these conditions. Four species of sea anemones were found belonging to the families Actiniidae and Aiptasiidae, and images from living specimens and cnidae are provided. Bunodosoma cavernatum and Exaiptasia diaphana are here recorded for the first time from the Colombian Caribbean. An updated list of sea anemones in the Colombian Caribbean, now comprising 34 taxa (i.e., 21 species and 13 identified at supra-specific levels), is provided.
\end{abstract}

Key words: Actiniidae, Aiptasiidae, benthic invertebrates, colonization marginal, environments, turbidity.

\section{INTRODUCTION}

The Atlántico Department comprises a coastal area of the Colombian Caribbean of about 72 $\mathrm{km}$ coastline, which differs from other areas of the region due to influence from discharges of the Magdalena River. This is due to the fact that the Magdalena River is the main fluvial artery of Colombia that flows into the Caribbean Sea, and it is estimated $\sim 143,9 \times 10^{6} \mathrm{t}$ of sediments are dissolved into the sea each year (Restrepo et al. 2006, 2017). These local conditions imply that many of the organisms inhabiting the area are subject to substantial stress caused by high turbidity and resuspension rates that occur throughout most of the year. Therefore, this sector of the Colombian Caribbean is very important to understand the processes of settlement and colonization of marine invertebrate groups, including cnidarians which could be quite demanding in this respect of high sedimentation and turbidity.

The Atlantico Department is one of the least studied areas of the Colombian Caribbean in terms of its faunal biodiversity. Although the study of some marine invertebrates in Colombia has increased in the last few decades, the main group among cnidarians that have been researched is the stony corals (order Scleractinia) (e.g., Díaz et al. 1996, 2000, Cairns 1999, Reyes 2000, Cendales et al. 2002, Vega-Sequeda et al. 2008, Reyes et al. 2010, Flórez \& Santodomingo 
2010). A few other studies related to hydrozoan, scyphozoan and cubozoan medusae are known for the Atlántico Department (e.g., DuránFuentes et al. 2018), but research about the diversity, distribution, and the ecological traits of other cnidarians, such as the sea anemones (class Anthozoa, order Actiniaria), have not been carried out.

Sea anemones are among the benthic marine invertebrates that are commonly found in coastal intertidal zones and coral reefs worldwide. In the Colombian Caribbean, 21 species of sea anemones have been recorded so far, as well as 13 other species identified at supraspecific levels (i.e., genus, family, superfamily, or order) (Table I), comprising about the $28 \%$ of the total number of known species in the Caribbean Sea (González-Muñoz et al. 2012). Records of sea anemones in Colombia have been reported from Tayrona National Park in Santa Marta (RiemannZürneck 1972, Manjarrés 1977, 1978, Brattström 1980, Reyes \& Campo 1992, Barrios-Suárez et al. 2002), the Rosario Island (Manjarrés 1979), San Andres, Providencia and Santa Catalina Islands (Werding et al. 1981, SIBM 2020), Cartagena Bay (Daly \& den Hartog 2004), North-east (Flórez \& Sandomingo, 2010, Gracia et al. 2013) and South-western Colombian Caribbean (Dueñas et al. 2019) (Table I), but no previous records of sea anemones are known from the Atlántico Department.

In this study, we documented the occurrence of four species of sea anemones in the Atlántico Department, belonging to families Actiniidae Rafinesque, 1815 and Aiptasiidae Carlgren, 1924, and short diagnoses and images from living specimens and cnidae are provided. The species Bunodosoma cavernatum (Bosc, 1802) and Exaiptasia diaphana (Rapp, 1829) are here recorded for the first time in the Colombian Caribbean, and the geographic distribution ranges of Actinostella flosculifera (Le Sueur, 1817) and Bunodosoma granuliferum (Le Sueur, 1817) are extended to the Atlántico Department. These records increase the number of sea anemone known for the Colombian Caribbean to 34 taxa. This study seeks to document which species of sea anemones inhabit the Atlántico Department as one of the first steps to understand the physiological processes involved in their presence in potentially stressful environmental conditions.

\section{MATERIALS AND METHODS}

Observations and collections of specimens were carried out in two locations of the Atlántico Department, Colombia (Fig. 1) from November 2018 to May 2019: Puerto Velero (three stations).(1) PV1 $\left(10^{\circ} 56^{\prime} 41.9^{\prime \prime} \mathrm{N} 75^{\circ} 02^{\prime} 36.3^{\prime \prime} \mathrm{W}\right),(2) \mathrm{PV} 2$

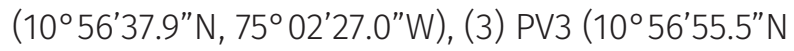
$75^{\circ} 02^{\prime} 09.8^{\prime \prime} \mathrm{W}$ ); and Caño Dulce Beach (two stations).- (1) CD1 (1056'33.0" N, 7501'35.3" W), (2) CD2 (10 $\left.56^{\prime} 10.2^{\prime \prime} \mathrm{N}, 75^{\circ} 01^{\prime} 44.7^{\prime \prime} \mathrm{W}\right)$. Collections were made on soft bottoms, rocky, and artificial substrates. The sea surface temperature in the area oscillates between 25.5 to $29.5{ }^{\circ} \mathrm{C}$, and the salinity between 34.4 to $37 \mathrm{ppm}$ (Posada \& Henao 2008). Two rainy seasons (from Abril to May and from October to November) and two dry seasons, from December to March and from July to September (Rangel-Buitrago et al. 2017), occur in the area. The influence of the Magdalena River on the department's coasts is evident, due to the presence of sediments as well as organic and inorganics materials (Rangel-Buitrago et al. 2017), with very low visibility of the water all year round.

specimens were collected by hand and snorkeling, using a small shovel, a hammer, and a chisel. Specimens were relaxed using $5 \% \mathrm{MgSO}_{4}$ seawater solution and subsequently fixed in $10 \%$ formalin seawater (Häussermann 
Table I. Previous and new records of actiniarian species in the Colombian Caribbean. Species are listed alphabetically by family and species. The symbol "*" indicate new records for the locality found in the present study, " $t$ " indicates new records for the Colombian Caribbean, and the citations of " $1-13$ " indicates localities of previously published records. Abbreviation: (SW) South-western and (NE) North-eastern Colombian Caribbean, (SAI) San Andrés Island and (PSCI) Providencia and Santa Catalina Islands. Citation: 1- Riemann-Zürneck 1972; 2Manjarrés 1977; 3- Manjarrés 1978; 4- Manjarrés 1979; 5- Brattström 1980; 6- Werding et al. 1981; 7- Reyes \& Campo 1992; 8- Barrios-Suárez et al. 2002; 9- Daly \& Den Hartog 2004; 10- Flórez \& Sandodomingo 2010; 11- Gracia et al. 2013; 12- Dueñas et al. 2019; 13- SIMB 2020.

\begin{tabular}{|c|c|c|c|c|c|c|c|c|c|c|c|c|c|c|c|c|c|c|}
\hline & \multicolumn{18}{|c|}{ Locality } \\
\hline Name taxa & 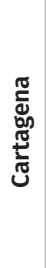 & 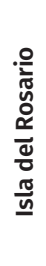 & 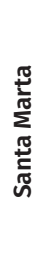 & 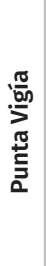 & 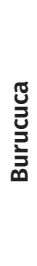 & 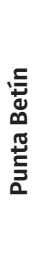 & 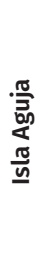 & 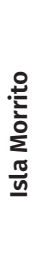 & 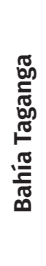 & 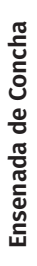 & 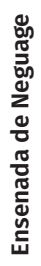 & 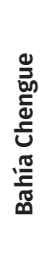 & n & 岂 & 㐫 & ড్ & 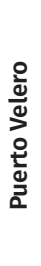 & 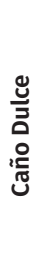 \\
\hline Actiniaria fam. gen. sp. & & & & & & & & & & & & & 12 & & & & & \\
\hline \multicolumn{19}{|l|}{ Family ACTINIIDAE Rafinesque, 1815} \\
\hline $\begin{array}{c}\text { Actinostella flosculifera (Le Sueur, 1817). (= P. } \\
\text { pretexta) }\end{array}$ & & 4 & & & & 3 & & & & 3 & 3 & & & & & & * & * \\
\hline Anthopleura texaensis (Carlgren \& Hedgpeth, 1952) & 9 & & & & & & & & & & & & & & & & & \\
\hline Isoaulactinia stelloides (McMurrich, 1889) & 9 & & & & & & & & & & & & & & & & & \\
\hline Bunodosoma cavernatum (Bosc, 1802) † & & & & & & & & & & & & & & & & & * & * \\
\hline Bunodosoma granuliferum (Le Sueur, 1817) & & & & & & 2 & & & & & 2 & & & & & 6 & * & * \\
\hline Condylactis gigantea (Weinland, 1860) & & 4 & 1 & 8 & & 2 & $\begin{array}{l}2 \\
8\end{array}$ & 2 & 2 & 2 & 2 & & & & 13 & 13 & & \\
\hline Leipsiceras pollens (McMurrich, 1898) & & & & & & & & & & & & & & 10 & & & & \\
\hline \multicolumn{19}{|l|}{ Family AIPTASIIDAE Carlgren, 1924} \\
\hline Aiptasia sp. Gosse, 1858 & & & & & & & & & & & & & & 11 & 13 & & & \\
\hline Bartholomea annulata (Le Sueur, 1817) & & 4 & 1 & 8 & & 2 & 8 & & 2 & 2 & 2 & & & & 13 & 13 & & \\
\hline Exaiptasia diaphana (Rapp, 1829) † & & & & & & & & & & & & & & & & & * & \\
\hline $\begin{array}{l}\text { Laviactis lucida (Duchassaing de Fombressin \& } \\
\text { Michelotti, 1860). (= H. lucida) }\end{array}$ & & 4 & & & & 3 & & & & 3 & & & & & & & & \\
\hline \multicolumn{19}{|l|}{ Family ALICIIDAE Duerden, 1895} \\
\hline Alicia sp. Johnson, 1861 & & & & 8 & & & 8 & & & & & & & & & & & \\
\hline Lebrunia coralligens (Wilson, 1890) & & 4 & & 8 & 3 & 3 & $\begin{array}{l}3 \\
8\end{array}$ & 3 & & 3 & 3 & & & & & 13 & & \\
\hline $\begin{array}{l}\text { Lebrunia neglecta Duchassaing \& Michelotti, } 1860 . \\
\qquad(=\text { L. danae })\end{array}$ & & 4 & & 8 & & 2 & $\begin{array}{l}2 \\
8\end{array}$ & & & & & & & & 13 & 13 & & \\
\hline Family ANDVAKIIDAE Danielssen, 1890 & & & & & & & & & & & & & & & & & & \\
\hline
\end{tabular}


Table I. Continuation.

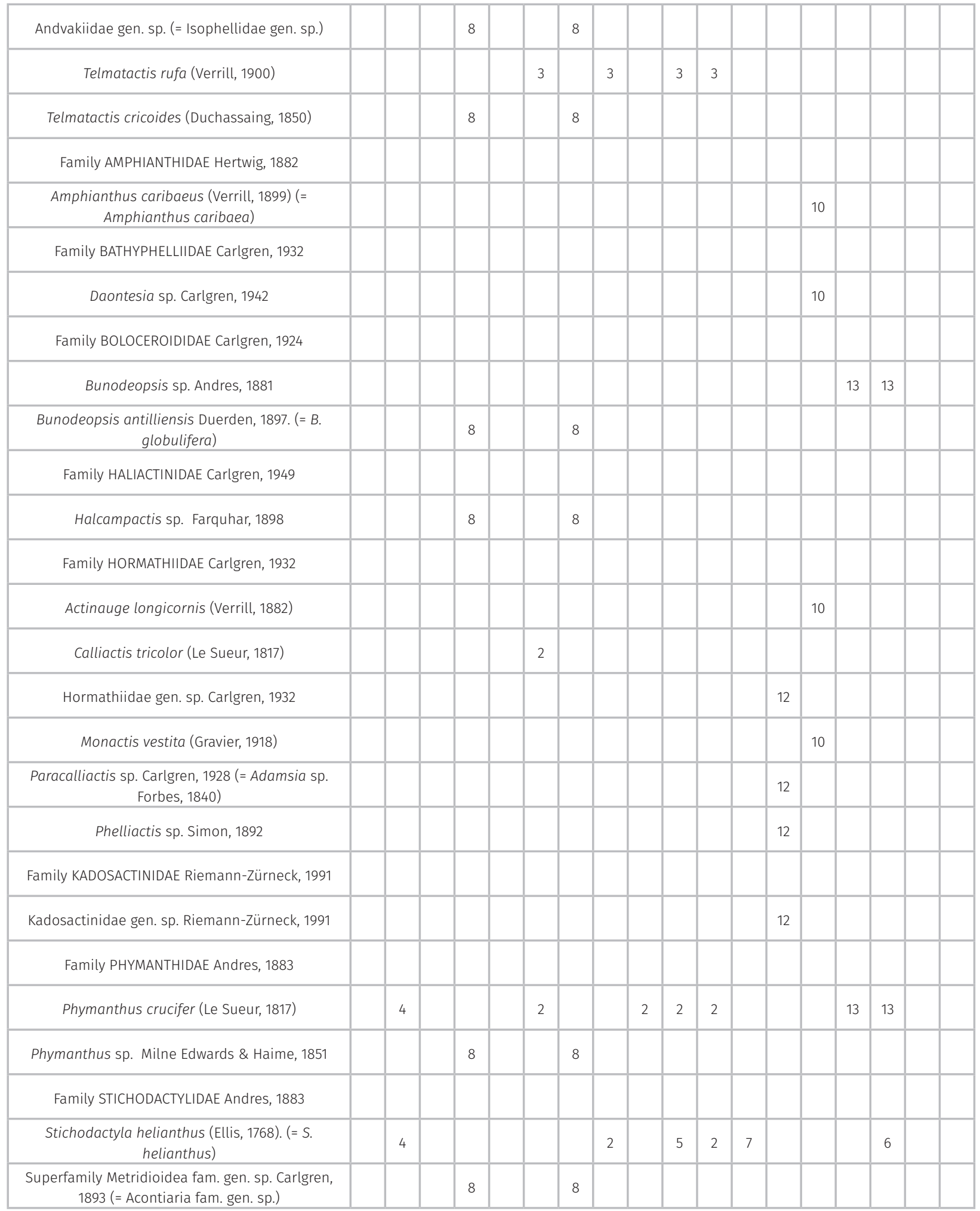


2004). External anatomy of specimens was examined made using a stereoscope (Leica EZ4). Measurements of specimens were obtained from live and relaxed specimens. Squash preparations of small amounts of tissue from tentacles, actinopharynx, filaments, column, acrorhagi, acontia, and marginal projections were made from two specimens for each species. Cnidae were examined using a light microscope Leica DM750 (1000x oil immersion) with adapted camera (Leica ICC50W), photographed and haphazardly measured. Cnidae terminology follows Östman (2000). Taxonomic classification follows Rodríguez et al. (2014). The synonym list for each species only contains a reference to the first citation of the species by a particular name. Voucher specimens were deposited in the collection of Marine Biodiversity of the Universidad of Atlántico, Colombia (registration code: UARC-CNI 061-072). Salinity and water transparency data were recorded from February to June 2019 using a salinometer (Pocket saltmeter ATAGO) and a Secchi disk (through horizontal visibility at a depth of $1 \mathrm{~m}$ ).

\section{RESULTS}

During the studied period (February to June 2019), the salinity measured in the sampled locations ranged from 27 to $28 \mathrm{ppm}$, and water transparency from 35 to $205 \mathrm{~cm}$ (Table II). A total of twelve specimens were collected, classified in two families, three genera, and four species.

\section{Taxonomic treatment}

Phylum Cnidaria Verrill, 1865

Class Anthozoa Ehrenberg, 1834

Subclass Hexacorallia Haeckel, 1900

Order Actiniaria Hertwig, 1882

Suborder Enthemonae Rodríguez \& Daly in Rodríguez et al. 2014

Superfamily Actinioidea Rafinesque, 1815

Family Actiniidae Rafinesque, 1815

Genus Actinostella Duchassaing, 1850

\section{Actinostella flosculifera (Le Sueur, 1817)}

(Figs. 2a-n)

Examined material: Puerto Velero Beach (PV3), two specimens (UARC-CNI 066, UARC-CNI 070).

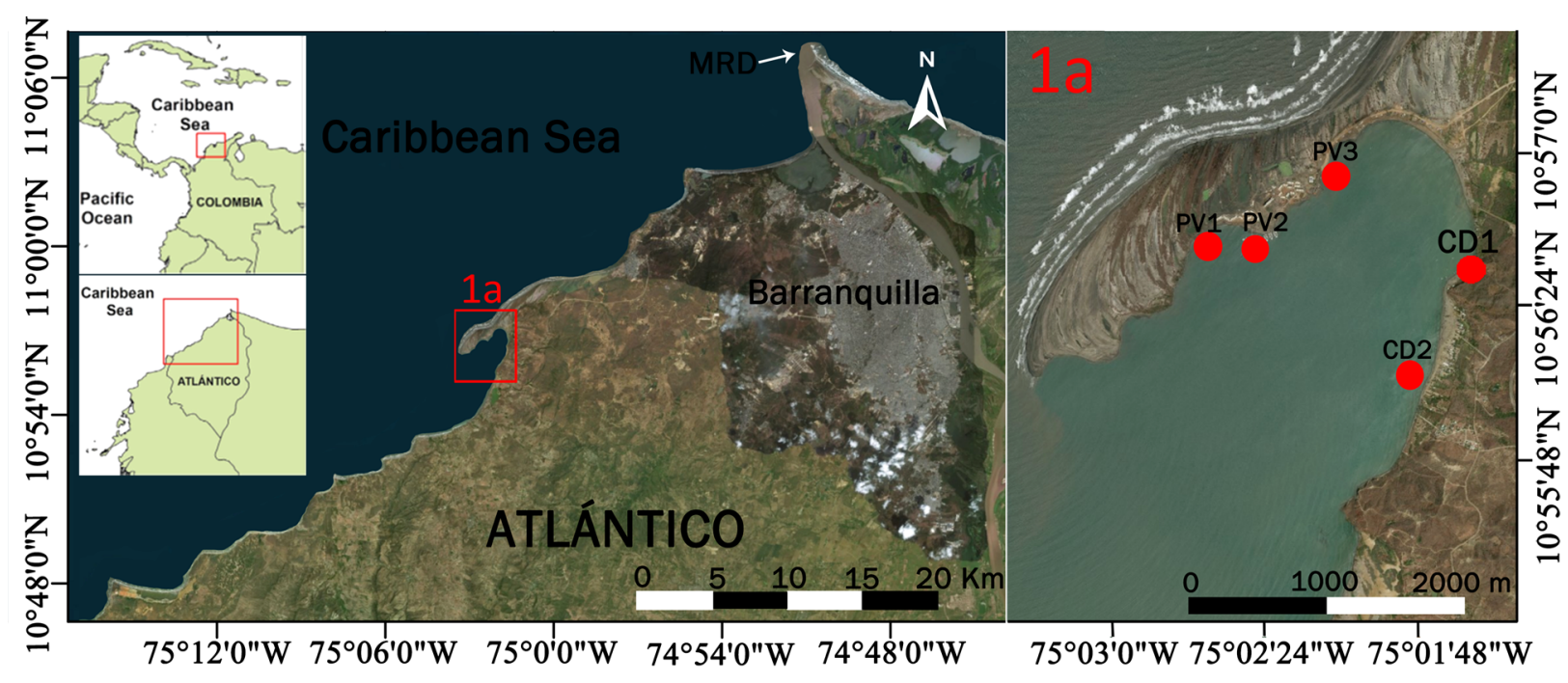

Figure 1. Map of the study area on the shallow shore of the Atlántico Department. 1a: Puerto Velero Bay, PV1 and PV2: Marina, PV3: Puerto Velero beach, CD1 and CD2: Caño Dulce beach. MRD: Magdalena River Delta. 
Table II. Recorded salinity and transparency of the water (Puerto Velero).

\begin{tabular}{|c|c|c|}
\hline Date & Salinity & $\begin{array}{c}\text { visibility of the } \\
\text { water }(\mathbf{c m})\end{array}$ \\
\hline February $21^{\text {st }}, 2019$ & 27 & $35-40$ \\
\hline March $17^{\text {th }}, 2019$ & 28 & $35-40$ \\
\hline March $27^{\text {th }}, 2019$ & 27 & $45-60$ \\
\hline May $11^{\text {th }}, 2019$ & 27.4 & $180-205$ \\
\hline May $18^{\text {th }}, 2019$ & 27.4 & $135-190$ \\
\hline Jun $29^{\text {th }}, 2019$ & 27 & 55 \\
\hline
\end{tabular}

\section{External anatomy}

Oral disc translucent pale-brown, often with white spots. Tentacles approximately 48 in number, hexamerously arranged in four cycles, smooth, conical, tapering distally, inner ones longer than outer ones, contractile, translucent pale-brown to light-pink color, with scattered small circular white spots. Column cylindrical to elongated, smooth but with small verrucae arranged in rows distally. Above verrucae lies a marginal ruff or collar with white and olivegreen color. Pedal disc well-developed. Pedal disc and column light-pink. Zooxanthellate.

Internal anatomy: See González-Muñoz et al. (2012).

Cnidae: basitrichs, long curved basitrichs, spirocysts, microbasic $b$ - and $p$-mastigophores (Figs. 2d-n; Table III).

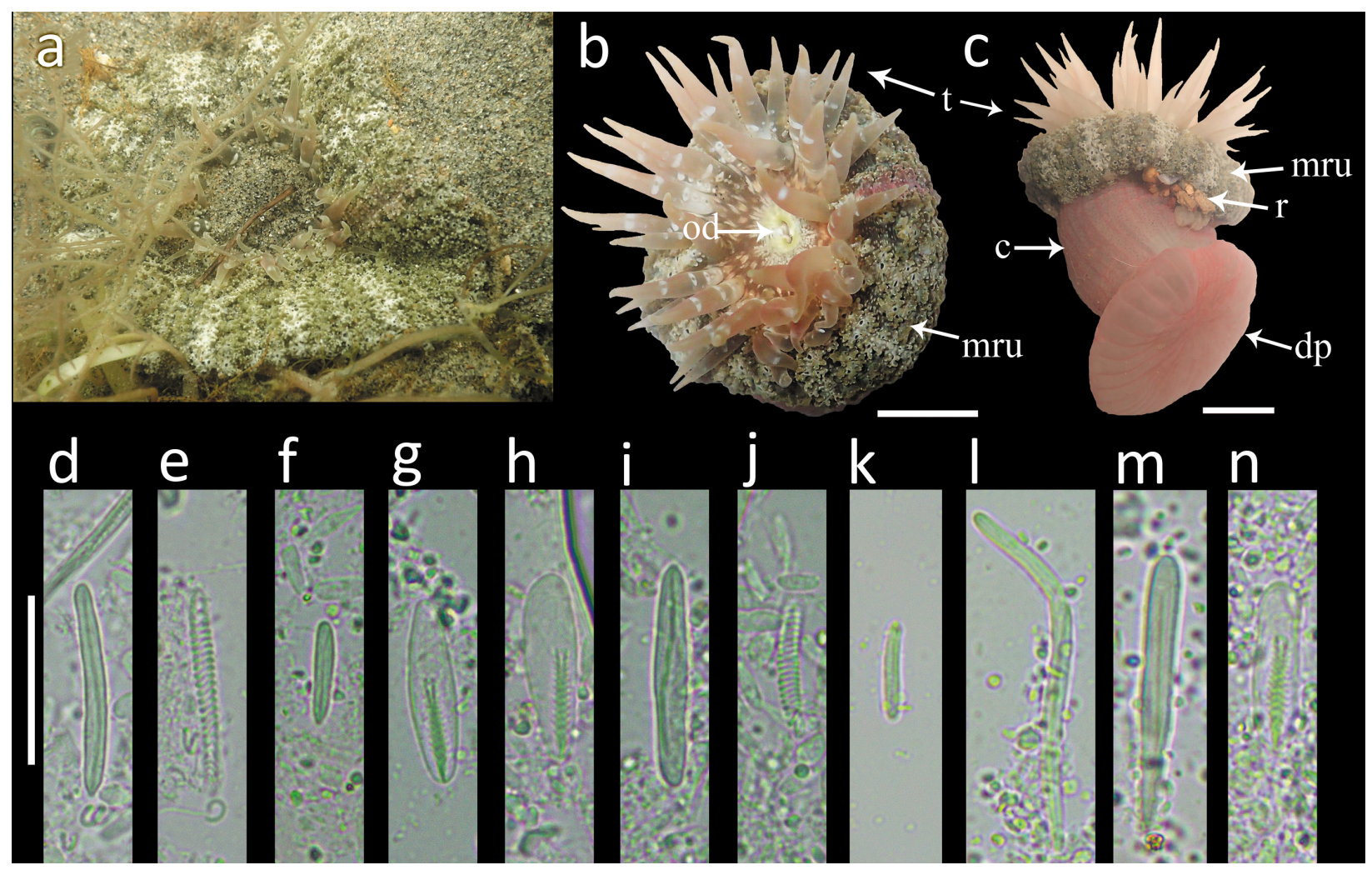

Figure 2. Actinostella flosculifera (Le Sueur, 1817). a live specimen in natural habitat. b oral view c lateral view. $\mathrm{d}-\mathrm{n}$ Cnidae. -tentacle: $\mathrm{d}$ basitrich e spirocyst; column: $\mathrm{f}$ basitrich $\mathrm{g}$ microbasic $p$-mastigophore; actinopharynx: $\mathrm{h}$ microbasic $p$-mastigophore i basitrich j spirocyst; filament: $\mathrm{k}$ small basitrich l long curved basitrich $\mathrm{m}$ microbasic b-mastigophore $\mathrm{n}$ microbasic $p$-mastigophore. Abbreviations. -c: column, mru: marginal ruff, od: oral disc, pd: pedal disc, r: rock, t: tentacle, vs: vesicles. Scale bars: b-c: $10 \mathrm{~mm}$; d-n: $20 \mu \mathrm{m}$. 
Table III. Size and distribution of cnidom in the sea anemones in the Atlántico Department. Abbreviation- al/ aw: average length/ average width; $\mathrm{dl} / \mathrm{dw}$ : standard density/ standard width; \#1 or \#2: number of cnidocysts in specimen 1 or 2; p: proportion.

\begin{tabular}{|c|c|c|c|c|c|c|c|c|c|c|c|}
\hline Species & Tissue & Cnidae & $\begin{array}{c}\text { Capsule } \\
\text { length (цm) } \\
\text { (min-max) }\end{array}$ & al & dl & $\begin{array}{c}\text { Capsule } \\
\text { width (цm) } \\
\text { (min-max) }\end{array}$ & aw & dw & $\begin{array}{l}\# \\
1\end{array}$ & $\begin{array}{l}\# \\
2\end{array}$ & $\mathbf{p}$ \\
\hline \multirow{13}{*}{$\begin{array}{l}\text { Actinostella } \\
\text { flosculifera }\end{array}$} & \multirow{3}{*}{ Tentacle } & Basitrich & $9.73-19.88$ & 17.03 & 3.24 & $1.79-2.69$ & 2.18 & 0.255 & 0 & 34 & $1 / 2$ \\
\hline & & Basitrich & $20.03-30.22$ & 24.39 & 2.69 & $1.86-3$ & 2.43 & 0.23 & 14 & 21 & $2 / 2$ \\
\hline & & Spirocyst & $7.71-25.68$ & 13.84 & 3.55 & $1.5-2.65$ & 2.14 & 0.29 & 34 & 35 & $2 / 2$ \\
\hline & \multirow{4}{*}{ Actinopharynx } & Basitrich & $9.53-12.1$ & 10.47 & 1.47 & $2.01-2.24$ & 2.12 & 0.11 & 3 & 16 & $2 / 2$ \\
\hline & & Basitrich & $16.41-30.43$ & 25.38 & 2.68 & $1.98-3.98$ & 3.06 & 0.42 & 0 & 36 & $1 / 2$ \\
\hline & & $\begin{array}{c}\text { Microbasic } \\
\text { p-mastigophore }\end{array}$ & $18.48-25.06$ & 21.47 & 1.93 & $4.63-7.32$ & 5.93 & 0.77 & 10 & 5 & $2 / 2$ \\
\hline & & Spirocyst & $2.17-16$ & 9.08 & 9.78 & $2.54-8.54$ & 5.53 & 4.23 & 2 & 0 & $2 / 2$ \\
\hline & \multirow[b]{2}{*}{ Column } & Basitrich & $7.21-15.16$ & 12.24 & 1.2 & $1.68-2.46$ & 2.06 & 0.18 & 35 & 35 & $2 / 2$ \\
\hline & & $\begin{array}{c}\text { Microbasic } \\
\text { p-mastigophore }\end{array}$ & $15.61-21.8$ & 19.39 & 2.1 & $3.9-6.08$ & 5.06 & 0.61 & 19 & 1 & $2 / 2$ \\
\hline & \multirow{4}{*}{ Filament } & Basitrich & $10.45-14.17$ & 12.33 & 1.04 & $1.6-2.62$ & 2.00 & 0.23 & 29 & 5 & $2 / 2$ \\
\hline & & $\begin{array}{l}\text { Long curve } \\
\text { basitrich }\end{array}$ & $29.02-42.02$ & 35.44 & 4.55 & $1.74-4.38$ & 2.80 & 0.98 & 0 & 5 & $1 / 2$ \\
\hline & & $\begin{array}{c}\text { Microbasic } \\
\text { b-mastigophore }\end{array}$ & $17.1-35.61$ & 32.63 & 4.00 & $2.57-4.14$ & 3.45 & 0.34 & 2 & 19 & $2 / 2$ \\
\hline & & $\begin{array}{c}\text { Microbasic } \\
\text { p-mastigophore }\end{array}$ & $16.78-24.63$ & 21.00 & 2.09 & $2.52-6.2$ & 4.38 & 0.92 & 32 & 13 & $2 / 2$ \\
\hline \multirow{14}{*}{$\begin{array}{c}\text { Bunodosoma } \\
\text { cavernatum }\end{array}$} & \multirow{3}{*}{ Tentacle } & Basitrich & $7.47-12.54$ & 9.92 & 2.17 & $1.29-2.53$ & 1.8 & 0.45 & 1 & 4 & $2 / 2$ \\
\hline & & Basitrich & $15.81-24.83$ & 19.42 & 1.39 & $1.83-2.88$ & 2.39 & 0.24 & 34 & 38 & $2 / 2$ \\
\hline & & Spirocyst & $8.26-21.37$ & 14.58 & 3.33 & $1.81-2.77$ & 2.28 & 0.39 & 16 & 32 & $2 / 2$ \\
\hline & \multirow{3}{*}{ Acrorhagi } & Basitrich & $6.85-22.52$ & 13.25 & 4.3 & $1.18-3.59$ & 2.29 & 0.58 & 34 & 29 & $2 / 2$ \\
\hline & & Spirocyst & $19.72-24.7$ & 21.12 & 2.09 & $2.47-3.61$ & 2.76 & 0.51 & 3 & 2 & $2 / 2$ \\
\hline & & Holotrich & $17.95-43.54$ & 33.41 & 7.25 & $3.15-5.82$ & 4.61 & 0.54 & 20 & 28 & $2 / 2$ \\
\hline & \multirow{3}{*}{ Actinopharynx } & Basitrich & $6.9-9.91$ & 8.3 & 1.13 & $1.33-2.17$ & 1.84 & 0.28 & 10 & 2 & $2 / 2$ \\
\hline & & Basitrich & $10.57-30.03$ & 19 & 5.52 & $1.73-4.42$ & 2.8 & 0.64 & 49 & 29 & $2 / 2$ \\
\hline & & $\begin{array}{c}\text { Microbasic } \\
\text { p-mastigophore }\end{array}$ & $16.06-18.89$ & 17.71 & 1.24 & $4.43-5.12$ & 4.76 & 0.3 & 2 & 2 & $2 / 2$ \\
\hline & \multirow{2}{*}{ Column } & Basitrich & $7.55-9.15$ & 8.16 & 1.2 & $1.37-2.65$ & 1.37 & 2.65 & 1 & 3 & $2 / 2$ \\
\hline & & Basitrich & $11.94-21.83$ & 15.17 & 2.39 & $1.81-4.5$ & 2.48 & 0.53 & 47 & 28 & $2 / 2$ \\
\hline & \multirow{3}{*}{ Filament } & Basitrich & $9.67-16.15$ & 12.78 & 1.89 & $1.6-3.01$ & 2.08 & 0.4 & 5 & 12 & $2 / 2$ \\
\hline & & $\begin{array}{c}\text { Microbasic } \\
\text { b-mastigophore }\end{array}$ & $27.7-40.58$ & 35.64 & 3.03 & $4.21-6.9$ & 5.74 & 0.62 & 30 & 11 & $2 / 2$ \\
\hline & & $\begin{array}{c}\text { Microbasic } \\
\text { p-mastigophore }\end{array}$ & $13.65-22.82$ & 19.44 & 1.33 & $2.81-6.6$ & 5.2 & 0.53 & 52 & 33 & $2 / 2$ \\
\hline
\end{tabular}


Table III. Continuation.

\begin{tabular}{|c|c|c|c|c|c|c|c|c|c|c|c|}
\hline \multirow{17}{*}{$\begin{array}{l}\text { Bunodosoma } \\
\text { granuliferum }\end{array}$} & \multirow{3}{*}{ Tentacle } & Basitrich & $9.34-14.81$ & 12.04 & 2.38 & $1.5-2.06$ & 1.81 & 0.28 & 3 & 1 & $2 / 2$ \\
\hline & & Basitrich & $18.93-33.96$ & 24.94 & 3.17 & $2.01-3.78$ & 2.64 & 0.33 & 28 & 31 & $2 / 2$ \\
\hline & & Spirocyst & $10.71-30.81$ & 18.47 & 5.17 & $1.96-3.56$ & 2.58 & 0.39 & 32 & 15 & $2 / 2$ \\
\hline & \multirow{3}{*}{ Acrorhagi } & Basitrich & $10.47-26.9$ & 18.79 & 3.97 & $1.86-3.69$ & 2.56 & 0.42 & 3 & 25 & $2 / 2$ \\
\hline & & Holotrich & $26.45-50.01$ & 40.37 & 4.81 & $2.64-5.37$ & 4.07 & 0.72 & 31 & 0 & $1 / 2$ \\
\hline & & Spirocyst & $13.82-20.63$ & 16.72 & 3.08 & $2.35-2.72$ & 2.55 & 0.16 & 29 & 1 & $2 / 2$ \\
\hline & \multirow{4}{*}{ Actinopharynx } & Basitrich & $11.26-16.7$ & 13.4 & 2.11 & $1.73-3.01$ & 2.24 & 0.47 & 1 & 4 & $2 / 2$ \\
\hline & & Basitrich & $18.45-29.3$ & 25.38 & 2.13 & $2.58-4.28$ & 3.46 & 0.39 & 30 & 28 & $2 / 2$ \\
\hline & & Spirocyst & $10.6-13.05$ & 11.63 & 1.27 & $2.04-3.02$ & 2.52 & 0.49 & 3 & 0 & $2 / 2$ \\
\hline & & $\begin{array}{c}\text { Microbasic } \\
\text { p-mastigophore }\end{array}$ & $17.73-20.44$ & 19.28 & 1.21 & $4.03-5.18$ & 4.58 & 0.57 & 4 & 0 & $2 / 2$ \\
\hline & \multirow{3}{*}{ Column } & Basitrich & $11.95-19.76$ & 16.29 & 2.08 & $1.8-3.35$ & 2.34 & 0.32 & 30 & 15 & $2 / 2$ \\
\hline & & Basitrich & $20.23-25.71$ & 23.12 & 2.1 & $2.11-3.75$ & 2.95 & 0.36 & 2 & 11 & $2 / 2$ \\
\hline & & Spirocyst & $15.88-21.23$ & 18.55 & 3.78 & $2.08-2.46$ & 2.27 & 0.27 & 2 & 0 & $2 / 2$ \\
\hline & \multirow{4}{*}{ Filament } & Basitrich & $10.09-14.26$ & 12.24 & 1.43 & $1.89-2.36$ & 2.14 & 0.18 & 6 & 2 & $2 / 2$ \\
\hline & & Basitrich & $22.52-25.59$ & 24.05 & 2.17 & $2.80-3.33$ & 3.07 & 0.36 & 1 & 1 & $2 / 2$ \\
\hline & & $\begin{array}{c}\text { Microbasic } \\
\text { b-mastigophore }\end{array}$ & $14.12-47.14$ & 31.99 & 9.1 & $2.85-8.93$ & 5.7 & 1.52 & 8 & 8 & $2 / 2$ \\
\hline & & $\begin{array}{c}\text { Microbasic } \\
\text { p-mastigophore }\end{array}$ & $12.14-23.62$ & 19.39 & 3.01 & $2.97-6.22$ & 4.49 & 0.78 & 29 & 29 & $2 / 2$ \\
\hline \multirow{13}{*}{$\begin{array}{l}\text { Exaiptasia } \\
\text { diaphana }\end{array}$} & \multirow{3}{*}{ Tentacle } & Basitrich & $12.16-19.08$ & 15.2 & 1.47 & $2.05-3.47$ & 2.55 & 0.32 & 30 & 30 & $2 / 2$ \\
\hline & & Spirocyst & $8.87-22.49$ & 14.12 & 3.26 & $1.72-3.89$ & 2.88 & 0.5 & 30 & 10 & $2 / 2$ \\
\hline & & $\begin{array}{c}\text { Microbasic } \\
\text { p-amastigophore }\end{array}$ & $20.36-32.6$ & 28.04 & 2.71 & $3.76-5.54$ & 4.79 & 0.41 & 30 & 8 & $2 / 2$ \\
\hline & \multirow{2}{*}{ Acontia } & Basitrich & $18.79-27.89$ & 23.47 & 2.01 & $2.16-3.53$ & 2.87 & 0.37 & 7 & 25 & $2 / 2$ \\
\hline & & $\begin{array}{c}\text { Microbasic } \\
\text { p-amastigophore }\end{array}$ & $26.97-69.3$ & 57.99 & 7.22 & $3.44-8.44$ & 6.18 & 1.09 & 18 & 23 & $2 / 2$ \\
\hline & \multirow{2}{*}{ Actinopharynx } & Basitrich & $10.63-21.89$ & 18.69 & 2.76 & $2.02-3.79$ & 2.90 & 0.43 & 18 & 8 & $2 / 2$ \\
\hline & & $\begin{array}{c}\text { Microbasic } \\
\text { p-amastigophore }\end{array}$ & $15.91-29.89$ & 25.02 & 2.13 & $3.17-5.95$ & 4.28 & 0.53 & 30 & 30 & $2 / 2$ \\
\hline & \multirow{3}{*}{ Filament } & Basitrich & $8.2-16.61$ & 12.26 & 2.07 & $1.61-3.25$ & 2.43 & 0.42 & 14 & 7 & $2 / 2$ \\
\hline & & $\begin{array}{c}\text { Microbasic } \\
\text { p-amastigophore }\end{array}$ & $9.76-18.36$ & 12.72 & 1.28 & $1.92-3.58$ & 2.64 & 0.30 & 30 & 22 & $2 / 2$ \\
\hline & & $\begin{array}{c}\text { Microbasic } \\
\text { p-amastigophore }\end{array}$ & $18.74-63.4$ & 31.65 & 15.29 & $2.88-6.35$ & 4.37 & 1.11 & 6 & 4 & $2 / 2$ \\
\hline & \multirow{3}{*}{ Column } & Basitrich & $6.81-15.5$ & 11.42 & 1.68 & $1.72-3.79$ & 2.68 & 0.50 & 30 & 30 & $2 / 2$ \\
\hline & & $\begin{array}{c}\text { Microbasic } \\
\text { p-amastigophore }\end{array}$ & $9.54-18.97$ & 16.38 & 1.61 & $2.06-5.22$ & 4.23 & 0.50 & 30 & 28 & $2 / 2$ \\
\hline & & $\begin{array}{c}\text { Microbasic } \\
\text { b-mastigophore }\end{array}$ & $10.93-15.98$ & 13.90 & 1.15 & $2.84-4.78$ & 4.10 & 0.33 & 26 & 26 & $2 / 2$ \\
\hline
\end{tabular}




\section{Size}

Fully expanded distal end up to $42.3 \mathrm{~mm}$ in diameter, oral disc about $14 \mathrm{~mm}$ in diameter, tentacles to $8.4-14.9 \mathrm{~mm}$ in length, column 24.4 $\mathrm{mm}$ in height and $21.4 \mathrm{~mm}$ in diameter, pedal disc to $23.2 \mathrm{~mm}$ in diameter.

\section{Natural history}

Actinostella flosculifera lives attached to buried rocks, in seagrass fields, between 0.1 to 5 m depths (González-Muñoz et al. 2012, 2016). Abundant zooxanthellae are found in the marginal ruff and tentacles. Häussermann (2004) reported that marginal ruff remains fully expanded during the day, allowing zooxanthellae to capture sunlight, but during the night, the tentacles remain expanded to capture food. In the Atlántico Department was found at $0.4 \mathrm{~m}$ depth, attached to rock surfaces.

\section{Distribution}

Along the entire Caribbean Sea, from Bermuda to Brazil, including the southern Gulf of Mexico (González-Muñoz et al. 2012, 2013, 2016); it has also been reported in the Canary Islands (Ocaña \& den Hartog 2002) and the Gulf of Guinea (Wirtz 2003). It has been previously reported in the Colombian Caribbean in Santa Marta (Punta Betín), Tayrona National Park (Concha Bay and Nenguange) (Manjarrés 1978) and Rosario Island, Cartagena (Manjarrés 1979) (Table I), but this is the first record from the Atlántico Department at Puerto Velero (PV3) and Caño Dulce beaches (CD1).

\section{Remarks}

Besides A. flosculifera, the species A. radiata (Duchassaing \& Michelotti 1860) and A. variabilis (Hargitt 1911) have also been recorded in the Caribbean Sea (González-Muñoz et al. 2012). Taxonomic information of $A$. radiata available is incomplete and thus prevents proper taxonomic comparisons with A. flosculifera. The specimens described as Cradactis variabilis (= Actinostella variabilis) by Hargitt (1911) actually belongs to the species Lebrunia coralligens (Wilson, 1890) (González-Muñoz et al. 2012). In addition to the cnidom of A. flosculifera reported by GonzálezMuñoz et al. (2012), in the Colombian material we found microbasic $p$-mastigophores in the column and in the filaments of two specimens, as well as spirocysts in the actinopharynx of one specimen.

Genus Bunodosoma Verrill, 1899

\section{Bunodosoma cavernatum (Bosc, 1802)}

(Figs. 3a-q)

\section{Examined material}

Caño Dulce Beach (CD1): one specimen (UARCCNI 068), Puerto Velero Beach (PV3): three specimens (UARC-CNI 063, 064, 067).

\section{External anatomy}

Oral disc smooth, olive-green with yellowish radial stripes (Fig. 3d). Tentacles approximately 96 in number, hexamerously arranged in five cycles, smooth, simple, conical, inner cycles longer than outer ones, contractile. Tentacles translucent, dark-blue, pale-brown or lightyellow, often with orange or light-red spots both partial (Figs. 3c and e) to total (Figs. 3a-b) on the back of the tentacle in the first three cycles, and pale-yellowish spots in the tentacles of the fourth cycle (Figs. 3a, c-e). Margin with 48 rounded marginal projections with acrorhagi (containing basitrichs and holotrichs) (Figs. 3b-c). Column cylindrical, densely covered with rounded dark vesicles arranged in longitudinal rows. Pedal disc well-developed (Fig. 3c). Column and pedal disc pale-orange (Fig. 3c) to pale-brown (Fig. 3d). Zooxanthellate.

Internal anatomy: See González-Muñoz et al. (2013). 


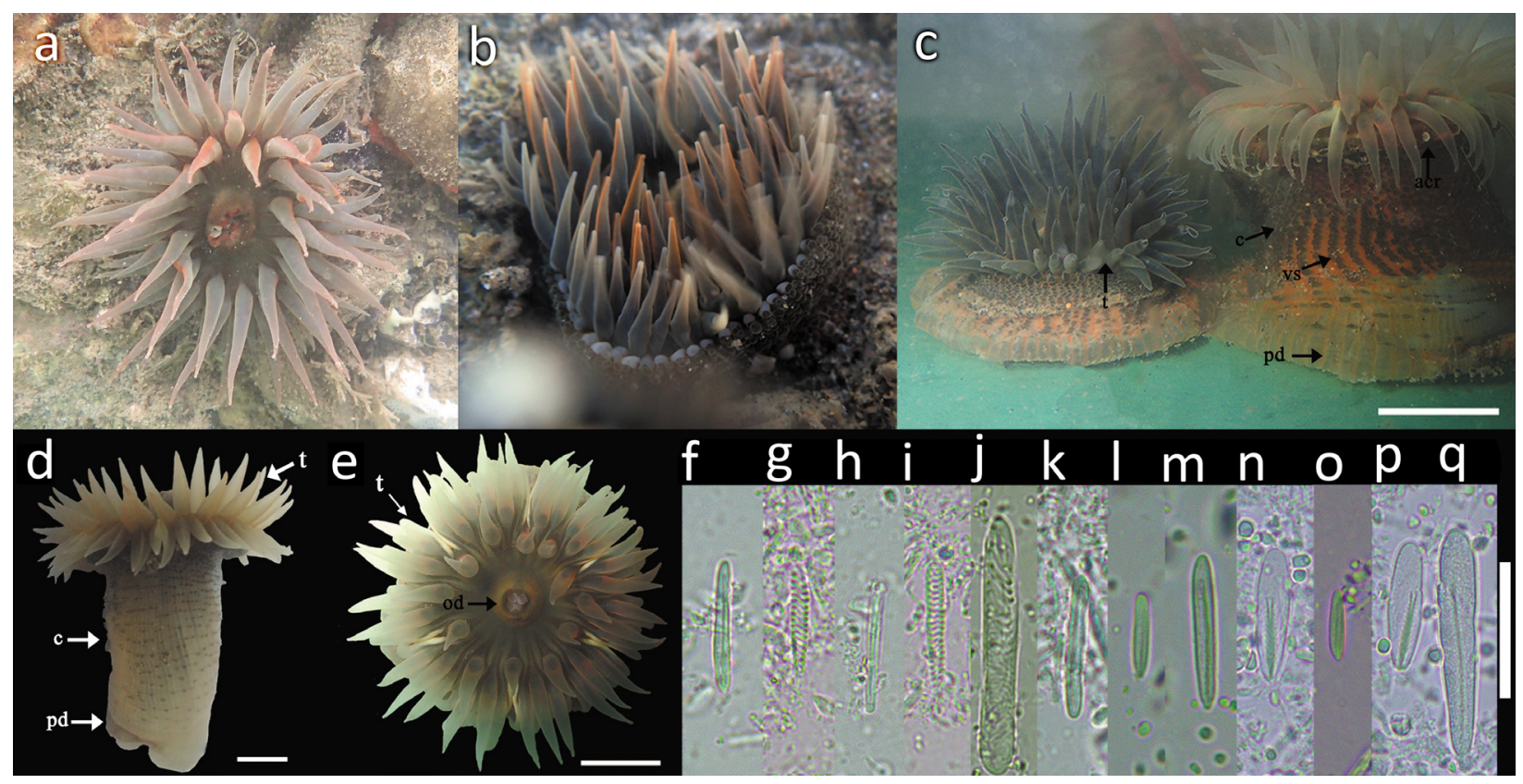

Figure 3. Bunodosoma cavernatum (Bosc, 1802). a-b Live specimen in natural habitat. c Lateral view of two different specimens. $d$ Lateral view. e Oral view. $f-q$ Cnidae. - tentacle: $f$ basitrich $g$ spirocyst; acrorhagi: $h$ basitrich i spirocyst $\mathrm{j}$ holotrich; column: $\mathrm{k}$ basitrich; actinopharynx: I small basitrich $\mathrm{m}$ basitrich $\mathrm{n}$ microbasic $p$-mastigophore; filament: o small basitrich $p$ microbasic $p$-mastigophore q microbasic $b$-mastigophore. Abbreviations. -acr: acrorhagi, c: column, od: oral disc, pd: pedal disc, t: tentacle, vs: vesicles. Scale bars: c-e: 10 $\mathrm{mm} ; \mathrm{f}-\mathrm{q}: 20 \mu \mathrm{m}$.

Cnidae: basitrichs, spirocysts, holotrichs, microbasic $b$ - and p-mastigophores (Figs. 3f-q; Table III).

\section{Size}

A fully expanded distal end to 54.5-56.9 mm in diameter, oral disc to $33.1 \mathrm{~mm}$ in diameter, tentacles to $7.3-17.5 \mathrm{~mm}$ in length, column to 19.4-39.5 $\mathrm{mm}$ in height and 20.4-22.9 $\mathrm{mm}$ in diameter, pedal disc to $15.5-22.1 \mathrm{~mm}$ in diameter.

\section{Natural history}

Bunodosoma cavernatum lives attached to rocky substrates at 0.5-7 m depth, in coral reefs, rocky shores, and seagrass fields (González-Muñoz et al. 2013, 2016). In the Atlántico Department it was found in the intertidal and subtidal zones, between 0.1 to $1.5 \mathrm{~m}$ depth, attached on rocks or metal sheets.

\section{Distribution}

Along the western Atlantic coast and the Caribbean Sea, from North Carolina to Barbados (González-Muñoz et al. 2013), and the Caroline Islands, Micronesia (Bosc 1802). This is the first record of $B$. cavernatum in the Colombian caribbean.

\section{Remarks}

Four valid species of genus Bunodosoma have been reported in the Caribbean Sea. Bunodosoma cavernatum and B. granuliferum (Le Sueur, 1817) mainly differ in their column color patterns, being beige, pale orange or reddish in the former, while the column of $B$. granuliferum presents a characteristic pattern of alternating light and dark longitudinal bands. The other two species, B. kuekenthali Pax, 1910 and B. sphaerulatum Durden, 1902, are currently considered valid species (Fautin 2016), although 
the distinction between these two species and B. cavernatum remains unclear and needs further revision.

\section{Bunodosoma granuliferum (Le Sueur, 1817)}

(Figs. 4a-s)

Examined material: Puerto Velero Beach (PV3): three specimens (UARC-CNI 061, 062, 069).

\section{External anatomy}

Oral disc smooth, flat, reddish-brown of green. Tentacles approximately 96 in number, hexamerously arranged in five cycles, smooth, simple, inner cycles longer than outer ones, contractile. Tentacles olive-green to greengreyish or light-yellow, often with white spots and flashes of purple or pink. Margin with 48 rounded marginal projections with acrorhagi (containing basitrichs and holotrichs). Column cylindrical, densely covered with rounded vesicles arranged in 24 alternating dark and light longitudinal bands (dark bands with about five rows of vesicles, light ones with about three). Pedal disc well-developed, olive-green to orange. Zooxanthellate.

Internal anatomy: See González-Muñoz et al. (2012).

Cnidae: basitrichs, spirocysts, holotrichs, microbasic $b$ - and $p$-mastigophores (Figs. 4e-s; Table III).

\section{Size}

Fully expanded distal end to $95.2 \mathrm{~mm}$ in diameter, oral disc to $46.1 \mathrm{~mm}$ in diameter, tentacles to 16.4-20.4 $\mathrm{mm}$ in length, column to $40.1 \mathrm{~mm}$ in height and $36.8 \mathrm{~mm}$ in diameter, pedal disc to $21.4 \mathrm{~mm}$ in diameter.

\section{Natural history}

Bunodosoma granuliferum lives attached to rocky substrates between 0.5 and $6 \mathrm{~m}$ depth,

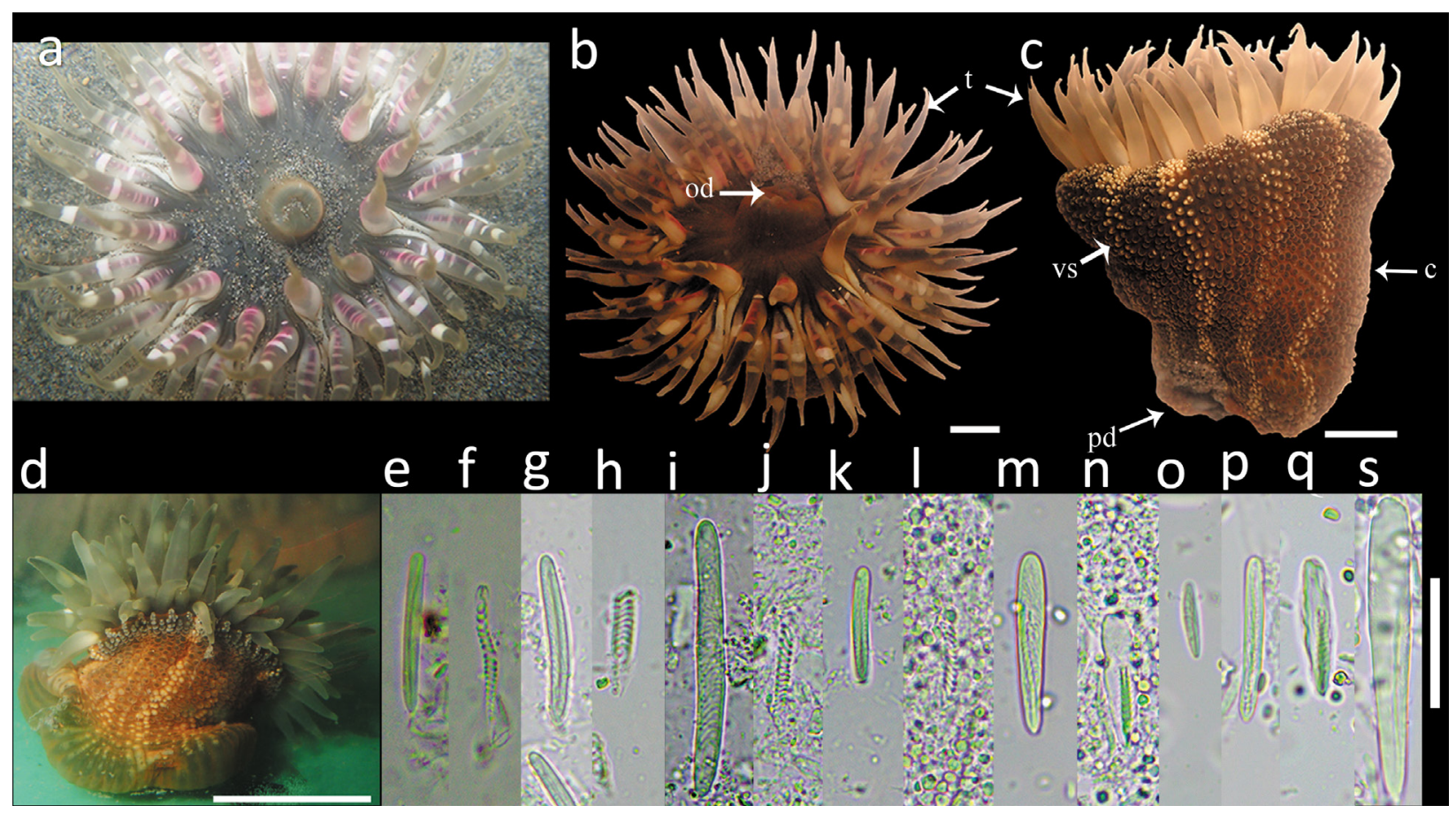

Figure 4. Bunodosoma granuliferum (Le Sueur, 1817). a live specimen in natural habitat. b oral view. c lateral view. d lateral view of the second specimen. e-s Cnidae. - tentacle: e basitrich $\mathrm{f}$ spirocyst; acrorhagi: $\mathrm{g}$ basitrich $\mathrm{h}$ spirocyst i holotrich; column: j spirocyst k basitrich; actinopharynx: I spirocyst $\mathrm{m}$ basitrich $\mathrm{n}$ microbasic $\mathrm{p}$-mastigophore; filament: o small basitrich $\mathrm{p}$ basitrich q microbasic $p$-mastigophore $s$ microbasic $b$-mastigophore. Abbreviations. -c: column, od: oral disc, pd: pedal disc, t: tentacle, vs: vesicles. Scale bars: b-d: $10 \mathrm{~mm}$; e-s: $20 \mu \mathrm{m}$. 
in coral reefs, rocky shores, and seagrasses fields (González-Muñoz et al. 2012, 2016). In the Atlántico Department, it was found in the intertidal and subtidal zones at depths between 0.5 and $1.5 \mathrm{~m}$, attached on rocks or metal sheets.

\section{Distribution}

Along the entire Caribbean Sea, from Bermuda to Barbados, including the southern Gulf of Mexico (González-Muñoz et al. 2012, 2013, 2015, 2016). It has been previously reported in the Colombian Caribbean in San Andres, Providencia and Santa Catalina Islands (Werding et al. 1981, SIBM 2020), and Punta Betín and Nenguange (Manjarrés 1977), but this is the first record of B. granuliferum in the Atlántico Department at Puerto Velero (PV1 and PV3) and Caño Dulce beaches (CD1 and CD2).

\section{Remarks}

Bunodosoma granuliferum differs from $B$. cavernatum by the column coloration pattern of alternating pale and dark longitudinal bands. Like González-Muñoz et al. (2012), we found spirocysts in the tentacles of the specimens examined, but also in the acrorhagi, filaments and actinopharynx. Holotrichs were observed only in one of the two specimens examined.

Superfamily Metridioidea Carlgren, 1893

Family Aiptasiidae Carlgren, 1924

Genus Exaiptasia Grajales \& Rodríguez, 2014

Exaiptasia diaphana (Rapp, 1829)

(Figs. 5 a-p)

Examined material: Puerto Velero Beach (PV2), one juvenile specimen (UACR-CNI 065), and two adult specimens (UARC-CNI 075).

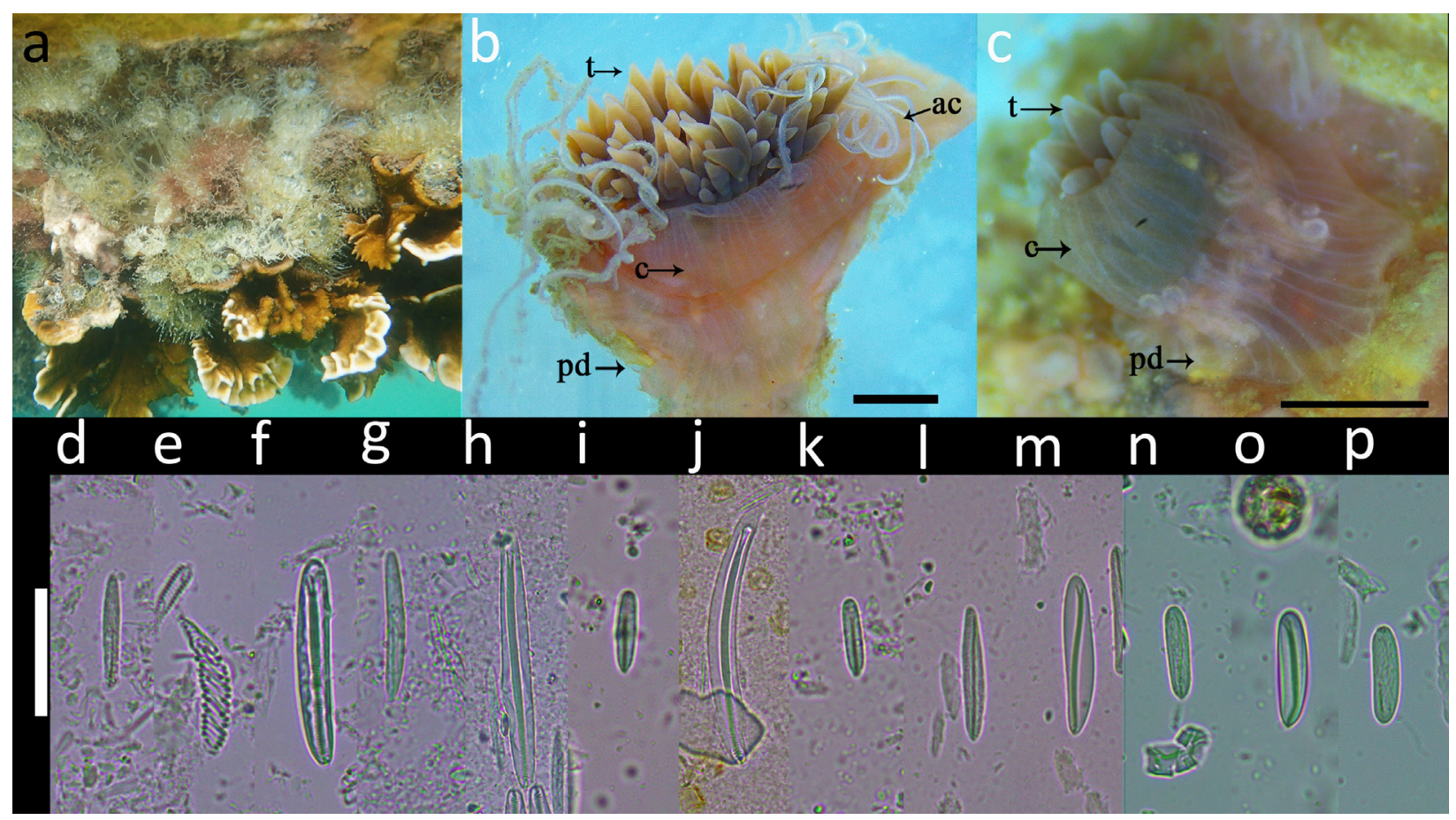

Figure 5. Exaiptasia diaphana (Rapp, 1829). a live specimen in natural habitat. b lateral view. c young anemone on the adult column (b). $d$-p Cnidae. - tentacle: $d$ basitrich e spirocyst $f$ microbasic $p$-amastigophore; acontia: $\mathrm{g}$ basitrich $\mathrm{h}$ large microbasic $p$-amastigophore; filament: i basitrich j large microbasic $p$-amastigophore $\mathrm{k}$ microbasic $p$-amastigophore; actinopharynx: I basitrich m microbasic $p$-amastigophore; column: $\mathrm{n}$ basitrich $\mathrm{o}$ microbasic $p$-amastigophore p microbasic $b$-mastigophore. Abbreviations. -ac: acontia, c: column, t: tentacle, pd: pedal disc. Scale bars: b: 2 mm; c: 1 mm; d-g, i, k-p: $20 \mu \mathrm{m}$; h, j: $30 \mu \mathrm{m}$. 


\section{External anatomy}

Oral disc smooth, flat, brown or green. Tentacles 48 in number, hexamerously arranged in four cycles, simple, smooth, inner cycles longer than outer ones, contractile. Tentacles pale-orange to greyish or light-yellow, often with white spots. Column cylindrical, smooth, translucent lightpink. Pedal disc well-developed, translucent light-pink. White acontia protruding from cinclides and the mouth (containing basitrichs and large microbasic p-amastigophores). Zooxanthellate.

Internal anatomy: See González-Muñoz et al. (2012) and Grajales \& Rodríguez (2014).

Cnidae: Basitrichs, spirocysts, microbasic $b$-mastigophores and $p$-amastigophores (Figs. 4d-p; Table III)

\section{Size}

Fully expanded distal end to 30.6-47.3 $\mathrm{mm}$ in diameter, oral disc to $12.3-28.6 \mathrm{~mm}$ in diameter, tentacles to $6.2-14.5 \mathrm{~mm}$ in length, column to $7.1-$ $15.01 \mathrm{~mm}$ in height and 16.8-27.9 $\mathrm{mm}$ in diameter, pedal disc to $10.4-12.1 \mathrm{~mm}$ in diameter.

\section{Natural history}

Exaiptasia diaphana is a tropical and subtropical shallow-water species, preferring calm and protected waters, found between 0 to $5 \mathrm{~m}$ depth (Grajales \& Rodríguez 2014). In the Atlántico Department, it was observed at 0.5-2 m depth covering artificial substrates as concrete, rope and metal sheets, as well as on some corals, sponges, and ascidians.

\section{Distribution}

Exaiptasia diaphana is a widespread species recorded worldwide along the northwestern Atlantic coast (Fautin 2013), Gulf of Mexico (González-Muñoz et al. 2013), Caribbean Sea (González-Muñoz et al. 2012), the coast of Brazil in the southwestern Atlantic Ocean (Zamponi et al. 1998, Farrapeira et al. 2007), Galapagos Islands (Fautin et al. 2007), and Australia (Grajales \& Rodríguez 2014). Grajales \& Rodríguez (2014) extended the distribution of the species to the Mediterranean Sea, western Africa, east and west Pacific coasts, Japan, and Hawaii. This is the first record of E. diaphana in the Colombian Caribbean.

\section{Remarks}

This species was formerly known as Exaiptasia pallida (Grajales \& Rodríguez 2014), but according to the ruling of the International Commission on Zoological Nomenclature (ICZN), the epithet diaphana retains priority over pallida and should be used as the name for this species (ICZN 2017). Criales (1984) mention E. diaphana (as Aiptasia pallida) as one of the sea anemone hosts species that could be found associated to the cleaner shrimp Periclimenes pedersoni Chace, 1958. However, Criales (1984) did not include this species in her list of sea anemones hosts found in the Santa Marta region of Colombia (Criales 1984). Thus, it is our interpretation that Criales (1984) did not observe this sea anemone species in the 1984 study, and thus we here document the first record of E. diaphana in the Colombian caribbean.

\section{DISCUSSION}

The new records of $B$. cavernatum and $E$. diaphana increase to 21 the number of sea anemone species for the Colombian Caribbean. These are not all the species found in the area but are those for which there are no unresolved taxonomic problems. There are at least other 13 actiniarian species that have been reported in the region but have been identified only to genus (8), family (3), superfamily (1) or to order (1) (Table I). Barrios-Suárez et al. (2002) 
documented four corallimorpharians and eleven actiniarians, including Alicia sp., Halcampactis sp., and Phymanthus sp., as well as a member of family Isophellidae (synonymized with family Andvakiidae by Rodríguez et al. 2012), and a member of superfamily Acontiaria (currently within superfamily Metridioidea since Rodríguez et al. 2014). Flórez \& Santodomingo (2010) reported four species and one genus at a depths of 200 to $500 \mathrm{~m}$ (Leipsiceras pollens (McMurrich, 1898), Amphianthus caribaeus (Verrill, 1899) (= Amphianthus caribaea), Daontesia sp. Carlgren, 1942, Actinauge longicornis (Verrill, 1882) and Monactis vestita (Gravier, 1918)). Gracia et al. (2013) reported two morpho-species of the genus Aiptasia on the La Guajira gas platform. Recently, Dueñas et al. (2019) reported Adamsia sp. (synonymized with genus Paracalliactis by Gusmão et al. 2019) and Phelliactis sp., as well as members of families Hormathiidae and Kadosactinidae, and another Actiniaria, from deep-sea bottoms in the south-western Colombian Caribbean.

The effect of the high turbidity and low salinity rates on the diversity of shallow-water sea anemones is still unclear, but appears that some species are able to tolerate stressful conditions. For example, Pereira et al. (2003) reported the species Anemonia sargassensis Hargitt, 1908 living on artificial substrates (breakwaters) at Casa Caiada Beach (Brazil), with a density of 20 ind $/ \mathrm{m}^{2}$, water transparency between 1 to $1.5 \mathrm{~m}$, suspended particulate material of $0.016 \mathrm{~g} / \mathrm{L}$, and salinity between 34.3 to $36.6 \mathrm{ppm}$. Moreover, Liu et al. (2015) used mesocosm experiments to simulate environments with nutrient enrichment (nitrate, phosphate and ammonium), high levels of turbidity (10-15 NTU), sedimentation (0.11-0.27 $\mathrm{g} / \mathrm{L}$ ) and salinity of $33 \mathrm{ppm}$, and they found that the sea anemone Mesactinia ganesis England, 1987 tolerated these conditions and was even able to attack corals as Acropora muricata (Linnaeus, 1758), causing their death.

In the Atlántico Department, E. diaphana was found in high densities (about $203 \mathrm{ind} / \mathrm{m}^{2}$ ) and abundances ranging from 74 to 524 individuals, covering artificial substrates. This could be related to the diverse reproductive strategies that E. diaphana exhibits, and the effects of environmental conditions on its asexual (pedal laceration) and sexual reproduction (sexual plasticity and interclonal fertilization), which allows it to successfully invade artificial marine substrates (Schlesinger et al. 2010). On the other hand, it has been suggested that a combined effect of variables (i.e., short-term sedimentation and nutrient enrichment) could cause replacement of corals by anemones on some studied coral reefs (Liu et al. 2015). In such a case, reproduction strategies could play an important role, due to the fact that sea anemones tend to increase their asexual reproduction rates under conditions of high nutrient exposure (Liu et al. 2015).

Although our results suggest that these species can tolerate or even benefit (e.g., E. diaphana) from the seemingly stressful conditions of the Magdalena River discharges, more studies are necessary to understand the physiological processes involved in their presence under these environments, as well as to determine the current diversity of sea anemones inhabiting the area.

\section{Acknowledgments}

We thank Dr. Nelson Rangel-Buitrago and Yully Contreras for assistance with some field trips and Dr. James D. Reimer (University of the Ryukyus, Japan) for his contributions and improvements to the document. This work is a contribution of the research group "Geology, Geophysics, and Marine - Coastal Process," Universidad del Atlántico (Barranquilla, Colombia). 


\section{REFERENCES}

BARRIOS-SUÁREZ LM, REYES JO, NAVAS GR \& GARCÍA CB. 2002. Distribution of anemones (Anthozoa: Actiniaria and Corallimorpharia) in the area of Santa Marta, Colombian Caribbean. Cienc Mar 28: 37-48.

BOSC LAG. 1802. Historie Naturalle des Vers. Paris, Chez Deterville, France, p. 300.

BRATTSTRÖM H. 1980. Rocky-shore zonation in the Santa Marta area, Colombia. Sarsia 65: 163-226.

CAIRNS SD. 1999. Species richness of recent Scleractinia. NMNH, Smithsonian Institution. Washington DC, U.S.A.

CENDALES MH, ZEA S \& DÍAZ JM. 2002. Geomorfología y unidades ecológicas del complejo de arrecifes de las Islas del Rosario e Isla Barú (Mar Caribe, Colombia). Rev Acad Colomb Cienc 26: 497-510.

CRIALES MM. 1984. Shrimps associated with coelenterates, echinoderms, and molluscs in the Santa Marta region, Colombia. J Crustacean Biol 4: 307-317.

DALY M \& DEN HARTOG JC. 2004. Anthopleura (Cnidaria: Anthozoa: Actiniaria) from the Gulf of Mexico and Caribbean. B Mar Sci 74: 401-421.

díAZ JM, SÁNCHEZ JA \& DÍAZ-PULIDO G. 1996. Geomorfología y formaciones arrecifales recientes de Isla Fuerte y Bajo Bushnell, plataforma continental del Caribe colombiano. Bol Invest Mar Cost 25: 87-105.

DÍAZ JM, BARRIOS LM, CENDALES MH, GARZÓN-FERREIRA J, GEISTER J, LÓPEZ-VICTORIA M, OSPINA GH, PARRA-VELANDIA F, PINZÓN J, VARGAS-ANGEL B, ZAPATA FA \& ZEA S. 2000. Áreas coralinas de Colombia. Santa Marta: INVEMAR, 176 p.

DUEÑAS L, CEDEÑO-POSSO C, GRAJALES A, HERRERA H, RODRÍGUEZ E, SÁNCHEZ JA, LEÓN J \& PUENTES V. 2019. First visual occurrence data for deep-sea cnidarians in the South-western Colombian Caribbean. Biodivers Data J 7: e33091.

DURÁN-FUENTES J, GRACIA A, OSORIO C \& CEDEÑO-POSSO C. 2018. Aporte al conocimiento de las medusas (Cnidaria: Medusozoa) en el departamento del Atlántico, Colombia. Rev Acad Colomb Cienc 42: 49-57.

FARRAPEIRA C, MELO A, BARBOSA DF \& SILVA K. 2007. Ship hull fouling in the Port of Recife, Pernambuco. Braz J Oceanogr 55: 207-221.

FAUTIN DG, CLEVELAND P, HICKMAN JR, DALY M \& MOLODTSOVA T. 2007. Shallow-water sea anemones (Cnidaria: Anthozoa: Actiniaria) and tube anemones (Cnidaria: Anthozoa: Ceriantharia) of the Galápagos Islands. Pac Sci 61: 549-573.
FAUTIN DG. 2013. Hexacorallians of the World. http:// geoportal.kgs.ku.edu/hexacoral/anemone2/index.cfm. Accessed 15 December 2018.

FAUTIN DG. 2016. Catalog to families, genera, and species of orders Actiniaria and Corallimorpharia (Cnidaria: Anthozoa). Zootaxa 4145: 1-449.

FLÓREZ P \& SANTOdOMINGO N. 2010. Cnidaria: Corales escleractinios, antipatarios, anémonas, zoantídeos, octocorales e hidroides. In: INVEMAR (Eds), Biodiversidad del margen continental del Caribe colombiano. Santa Marta: INVEMAR, p. 151-173.

GONZÁLEZ-MUÑOZ R, SIMÕES N, SÁNCHEZ-RODRÍGUEZ J, RODRÍGUEZ E \& SEGURA-PUERTAS L. 2012. First inventory of sea anemones (Cnidaria: Actiniaria) of the Mexican Caribbean. Zootaxa 3556: 1-38.

GONZÁLEZ-MUÑOZ R, SIMÕES N, TELLO-MUSI JL \& RODRÍGUEZ E. 2013. Sea anemones (Cnidaria, Anthozoa, Actiniaria) from coral reefs in the southern Gulf of Mexico. ZooKeys 341: 77-106.

GONZÁLEZ-MUÑOZ R, TELLO-MUSI JL \& SIMÕES N. 2015. LaS anémonas del Sistema Arrecifal Veracruzano. In: Granados-Barba A, Ortiz-Lozano LD, Salas-Monreal D \& González-Gándara C (Eds), Aportes al conocimiento del Sistema Arrecifal Veracruzano: hacia el corredor arrecifal del Suroeste del Golfo de México, $1^{\text {st }}$ ed., San Francisco de Campeche: Universidad Autónoma de Campeche, p. 101-118.

GONZÁLEZ-MUÑOZ R ET AL. 2016. Sea anemones (Cnidaria: Actiniaria, Corallimorpharia, Ceriantharia, Zoanthidea) from marine shallow-water environments in Venezuela: new records and an updated inventory. Mar Biodivers Rec 9: 18.

GRACIA A, CRUZ N, BORRERO G, BÁEZ DP \& SANTODOMINGO N. 2013. Invertebrados marinos asociados con las plataformas de gas en La Guajira (Caribe Colombiano). Bol Invest Mar Cost 42: 361-386.

GRAJALES A \& RODRíGUEZ E. 2014. Morphological revision of the genus Aiptasia and the family Aiptasiidae (Cnidaria, Actiniaria, Metridioidea). Zootaxa 3826: 55-100.

GUSMÃO LC RODRÍGUEZ E \& DALY M. 2019. Description of Calliactis tigris sp. nov.: reconciling taxonomy and phylogeny in hermit-crab symbiotic anemones (Cnidaria: Actiniaria: Hormathiidae). Org Divers Evol 1-17.

HÄUSSERMANN V. 2004. Identification and taxonomy of soft-bodied hexacorals exemplified by Chilean sea anemones; including guidelines for sampling, preservation and examination. J Mar Biol Assoc UK 84: 931-936. 
HARGITT CW. 1911. Cradactis variabilis: an apparently new Tortugan actinian. Carnegie Instit Wash 3: 51-53.

ICZN. 2017. Opinion 2404 (Case 3633) - Dysactis pallida Agassiz in Verrill, 1864 (currently Aiptasia pallida; Cnidaria, Anthozoa, Hexacorallia, Actiniaria): precedence over Aiptasia diaphana (Rapp, 1829), Aiptasia tagetes (Duchassaing de Fombressin and Michelotti, 1864), Aiptasia mimosa (Duchassaing de Fombressin \& Michelotti, 1864) and Aiptasia inula (Duchassaing de Fombressin \& Michelotti, 1864) not approved. Bull Zool Nomencl 74: 130-132.

LIU PJ, HSIN MC, HUANG YH, FAN TY, MENG PJ, LU CC \& LIN HJ. 2015. Nutrient enrichment coupled with sedimentation favors sea anemones over corals. Plos One 10: e0125175.

MANJARRÉS GA. 1977. Contribución al conocimiento de las Actinias en la región de Santa Marta. An Ins Invest Mar Punt Betín 9: 91-104.

MANJARRÉS GA. 1978. Nuevos encuentros de actinias (Hexacorallia) en la región de Santa Marta, Colombia. An Ins Invest Mar Punt Betín 10: 127-132.

MANJARRÉS GA. 1979. Observaciones sobre los Anthozoa (excepto Madreporaria) en las islas del Rosario, Santa Marta, Colombia. An Ins Invest Mar Punt Betín 11: 23-24.

OCAÑA Ó \& DEN HARTOG JC. 2002. A catalogue of actiniaria and corallimorpharia from the Canary Islands and from Madeira. Arquipélago 19: 33-54.

ÖSTMAN C. 2000. A guideline to nematocyst nomenclature and classification, and some notes on the systematic value of nematocysts. Scie Mar 64: 31-46.

PEREIRA LCC, JIMÉNEZ JA, GOMES PB, MEDEIROS C \& COSTA RAA. 2003. Effects of sedimentation on scleractinian and actinian species in artificial reefs at the Casa Caiada beach (Brazil). J Coastal Res 35: 418-425.

POSADA B \& HENAO W. 2008. Diagnóstico de la erosión en la zona costera del Caribe colombiano. Santa Marta: INVEMAR, 200 p.

RANGEL-BUITRAGO N, WILLIAMS A, ANFUSO G, ÁRIAS M \& GRACIA A. 2017. Magnitudes, sources, and management of beach litter along the Atlántico department coastline, Caribbean coast of Colombia. Ocean Coast Manag 138: 142-157.

RESTREPO JD, ZAPATA P, DÍAZ JM, GARZÓN-FERREIRA J \& GARCÍA CB. 2006. Fluvial fluxes into the Caribbean Sea and their impact on coastal ecosystems: The Magdalena River, Colombia. Glob Planet Change 50: 33-49.

RESTREPO JC, OREJARENA O \& TORREgROZA AC. 2017. Suspended sediment load in northwestern South
America (Colombia): A new view on variability and fluxes into the Caribbean Sea. J South Amer Earth Sci 80: 340-352.

REYES RR \& CAMPO N. 1992. Macroinvertebrados colonizadores de raíces de Rhizophora mangle en la Bahía de Chengue, Caribe colombiano. Bol Invest Mar Cost 21: 101-116.

REYES J. 2000. Lista de los corales (Cnidaria: Anthozoa: Scleractinia) de Colombia. Bio Col 1: 164-176.

REYES J, SANTODOMINGO N \& FLÓREZ P. 2010. Corales escleractinios de Colombia. Santa Marta: INVEMAR, 246 p.

RIEMANN-ZÜRNECK K. 1972. Über Aktinien aus der Karibik: Rhodactis, Condylactis, Bartholomea. Mitt. Inst ColomboAlemán Invest Cie 6: 73-76.

RODRÍGUEZE, BARBEITOS M, DALYM, GUSMAO LC \& HÄUSSERMANN V. 2012. Toward a natural classification: phylogeny of acontiate sea anemones (Cnidaria, Anthozoa, Actiniaria). Cladistics 28: 375-392.

RODRÍGUEZ E, BARBEITOS MS, BRUGLER MR, CROWLEY LM, GRAJALES A, GUSMÃO L, HÄUSSERMANN V, REFT A \& DALY M. 2014. Hidden among sea anemones: the first comprehensive phylogenetic reconstruction of the order Actiniaria (Cnidaria, Anthozoa, Hexacorallia) reveals a novel group of hexacorals. PLoS One 9: e96998.

SCHLESINGER A, KRAMARSKY-WINTER E, ROSENFELD H, ARMOZAZVOLONI R \& LOYA Y. 2010. Sexual plasticity and selffertilization in the sea anemone Aiptasia diaphana. PloS One 5: e11874.

SIBM. 2020. Sistema de Información sobre Biodiversidad Marina de Colombia. Instituto de Investigaciones Marinas y Costeras - INVEMAR. http://www.invemar.org. co/siam/sibm. Accessed 15 December 2020.

VEGA-SEQUEDA J, RODRÍGUEZ-RAMÍREZ A, REYES-NIVIA MC \& NAVAS-CAMACHO R. 2008. Formaciones coralinas del área de Santa Marta: estado y patrones de distribución espacial de la comunidad bentónica. Bol Invest Mar Cost 37: 87-105.

WERDING B, GARZÓN-FERREIRA L \& ZEA S. 1981. Informe sobre los resultados de la expedición Providencia I a las islas de Providencia y Santa catalina (Colombia). Ins Invest Mar Punt Betín.

WIRTZ P. 2003. New records of marine invertebrates from São Tomé Island (Gulf of Guinea). J Mar Biol Ass UK 83: 735-736.

ZAMPONI M, BELÉM M, SCHLENZE \& ACUÑA FH. 1998. Distribution and some ecological aspects of Corallimorpharia and 
Actiniaria from shallow waters of the South American Atlantic coasts. Physis 55: 31-45.

\section{How to cite}

DURÁN-FUENTES J, GRACIA A \& GONZÁLEZ-MUÑOZ R. 2021. Sea anemones (Cnidaria, Anthozoa, Actiniaria) in high sedimentation environments influenced by the Magdalena River (Colombian Caribbean). An Acad Bras Cienc 94: e20190862. DOI 10.1590/0001-3765202120190862.

Manuscript received on July 27, 2019;

accepted for publication on May 3, 2020

\section{JEFERSON DURÁN-FUENTES ${ }^{1}$}

https://orcid.org/0000-0001-6018-8699

\section{ADRIANA GRACIA ${ }^{1}$}

https://orcid.org/0000-0002-4771-5295

\section{RICARDO GONZÁLEZ-MUÑOZ}

https://orcid.org/0000-0002-9509-0269

${ }^{1}$ Universidad del Atlántico, Programa de Biología, Facultad de Ciencias Básicas, Km 7, 8-49, Puerto Colombia, Atlántico, Colombia

${ }^{2}$ Laboratorio de Biología de Cnidarios, Instituto de Investigaciones Marinas y Costeras, Facultad de Ciencias Exactas y Naturales, Universidad Nacional de Mar del PlataCONICET, Rodríguez Peña 4046, 7600 Mar del Plata, Argentina

Corresponding author: Jeferson Durán-Fuentes

E-mail: jalexisduran@mail.uniatlantico.edu.co

\section{Author contributions}

J.D. contributed to the design and implementation of the research, analysis and writing of the manuscript, and developed the study. A.G. contributed to the writing of the manuscript, analysis of the results, and developed the study. R.G-M. contributed to the writing and analysis of the document.

\section{(cc) BY}

